\title{
Symmetry indicators for inversion-symmetric non-Hermitian topological band structures
}

\author{
Vecsei, Pascal M ; Denner, M Michael ; Neupert, Titus ; Schindler, Frank
}

\begin{abstract}
We characterize non-Hermitian band structures by symmetry indicator topological invariants. Enabled by crystalline inversion symmetry, these indicators allow us to short-cut the calculation of conventional non-Hermitian topological invariants. In particular, we express the three-dimensional winding number of pointgapped non-Hermitian systems, which is defined as an integral over the whole Brillouin zone, in terms of symmetry eigenvalues at high-symmetry momenta. Furthermore, for time-reversal symmetric non-Hermitian topological insulators, we find that symmetry indicators characterize the associated Chern-Simons form, whose evaluation usually requires a computationally expensive choice of smooth gauge. In each case, we discuss the non-Hermitian surface states associated with nontrivial symmetry indicators.
\end{abstract}

DOI: https://doi.org/10.1103/PhysRevB.103.L201114

Posted at the Zurich Open Repository and Archive, University of Zurich ZORA URL: https://doi.org/10.5167/uzh-204507

Journal Article

Published Version

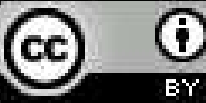

The following work is licensed under a Creative Commons: Attribution 4.0 International (CC BY 4.0) License.

Originally published at:

Vecsei, Pascal M; Denner, M Michael; Neupert, Titus; Schindler, Frank (2021). Symmetry indicators for inversionsymmetric non-Hermitian topological band structures. Physical review B, 103(20):L201114.

DOI: https://doi.org/10.1103/PhysRevB.103.L201114 


\title{
Symmetry indicators for inversion-symmetric non-Hermitian topological band structures
}

\author{
Pascal M. Vecsei $\odot,{ }^{1,}{ }^{*}$ M. Michael Denner $\odot,{ }^{1}$ Titus Neupert $\odot,{ }^{1}$ and Frank Schindler $\odot^{2}$ \\ ${ }^{1}$ Department of Physics, University of Zurich, Winterthurerstrasse 190, 8057 Zurich, Switzerland \\ ${ }^{2}$ Princeton Center for Theoretical Science, Princeton University, Princeton, New Jersey 08544, USA
}

(Received 23 March 2021; accepted 3 May 2021; published 21 May 2021)

\begin{abstract}
We characterize non-Hermitian band structures by symmetry indicator topological invariants. Enabled by crystalline inversion symmetry, these indicators allow us to short-cut the calculation of conventional non-Hermitian topological invariants. In particular, we express the three-dimensional winding number of point-gapped nonHermitian systems, which is defined as an integral over the whole Brillouin zone, in terms of symmetry eigenvalues at high-symmetry momenta. Furthermore, for time-reversal symmetric non-Hermitian topological insulators, we find that symmetry indicators characterize the associated Chern-Simons form, whose evaluation usually requires a computationally expensive choice of smooth gauge. In each case, we discuss the nonHermitian surface states associated with nontrivial symmetry indicators.
\end{abstract}

DOI: 10.1103/PhysRevB.103.L201114

Non-Hermitian topological band structures generalize the concept of crystalline band-structure topology to systems with loss and gain [1-5]. In a first approximation to open quantum systems in condensed matter, the Bloch description of crystals is adapted to accommodate non-Hermitian hopping matrices while remaining fundamentally a Hamiltonian-based single-particle description. The approximation breaks down on timescales comparable to the associated non-Hermitian decay lengths, but it provides insight into how well-known concepts from Hermitian band theory are modified to account for dissipation. In particular, it turns out that the celebrated bulk-boundary correspondence of topological insulators should be revisited [6-12]: the non-Hermitian skin effect [13-21] is a striking example of the sensitive dependence of (single-particle [22,23]) non-Hermitian systems on the boundary conditions. In the same tradition as many other topological features of electronic band structures, it has already been theoretically predicted and experimentally realized in a range of classical analog systems [24-29].

Nevertheless, the bulk classification of topological insulators with respect to (crystalline) symmetries can be adapted rather straightforwardly to non-Hermitian systems, as long as the notion of a bulk gap is specified [30-34]: for Hermitian insulators, the gap separates the occupied and empty band subspaces. For non-Hermitian systems, which in general have complex energy spectra, one distinguishes line gaps and point gaps. In the complex plane, a line gap separates the spectrum into two disconnected "bands," while a point gap constitutes a region (centered around $E=0$ without loss of generality) that is devoid of eigenstates. Only point-gapped systems are intrinsically non-Hermitian, in that they cannot be deformed to Hermitian systems without closing the gap.

A (partial) topological classification of point-gapped insulators has been achieved, but the corresponding topological

*Corresponding author: pascal.vecsei@aalto.fi invariants are often formulated in terms of computationally expensive Brillouin zone integrals $[30,31,35]$. For Hermitian systems, crystalline symmetries have often been fruitfully used to simplify the calculation of topological invariants of noncrystalline topological phases [36-41]. In this Letter, we show that the same is possible for non-Hermitian band structures: We first prove symmetry indicator formulas for the winding numbers of one-dimensional (1D) and three-dimensional (3D) non-Hermitian insulators in AltlandZirnbauer class A. Then, we formulate a symmetry-indicatorbased invariant for 3D point-gapped systems in symmetry class AII, where the usual invariant is especially costly in that it requires the choice of a smooth gauge over the Brillouin zone [42]. Our results point toward a unified understanding of Hermitian and non-Hermitian crystalline topology.

$1 D$ winding number. Consider the disorder-free HatanoNelson chain [13-15] with Hamiltonian

$$
H=\sum_{i} t_{\mathrm{r}} c_{i+1}^{\dagger} c_{i}+t_{1} c_{i}^{\dagger} c_{i+1},
$$

where $t_{\mathrm{r}} \neq t_{1}$ are the real-valued right and left hopping amplitudes, respectively, and $c_{i}^{\dagger}$ creates an electron at site $i \in$ $1, \ldots, L(L$ is the system size; we choose a lattice spacing $a=1$ ). With periodic boundary conditions (PBCs), the system is described by the $1 \times 1$ Bloch Hamiltonian

$$
\mathcal{H}(k)=t_{\mathrm{r}} e^{i k}+t_{1} e^{-i k},
$$

where $k=2 \pi / L, \ldots, 2 \pi$ is the crystal momentum. The spectrum then forms an ellipse in the complex plane, and the single-particle eigenstates are standard Bloch waves. Under the introduction of open boundary conditions (OBCs), the spectrum collapses onto the real line, while all eigenstates accumulate on only one edge of the system. For $\left|t_{\mathrm{r}}\right|>\left|t_{1}\right|$ this is the right edge, while for $\left|t_{\mathrm{r}}\right|<\left|t_{1}\right|$ this is the left edge. This distinctly non-Hermitian phenomenon of spectral collapse in OBCs is dubbed the non-Hermitian skin effect [6-9,13-21]. 
Importantly, the skin effect is an unavoidable property of all Bloch Hamiltonians that have a nontrivial 1D winding number (taking $L \rightarrow \infty$ )

$$
w_{1 \mathrm{D}}=\int_{0}^{2 \pi} \frac{d k}{2 \pi i} \frac{d}{d k} \ln \operatorname{det} \mathcal{H}(k) \in \mathbb{Z},
$$

which is well-defined in the presence of a point gap at $E=$ 0 [21]. For the Hamiltonian in Eq. (2), we obtain $w_{1 \mathrm{D}}=$ $\operatorname{sgn}\left(\left|t_{\mathrm{r}}\right|-\left|t_{1}\right|\right)$. In this sense, $w_{1 \mathrm{D}}$ is an integer-valued invariant of intrinsically 1D non-Hermitian topological phases that requires no symmetries for its quantization. (The topological classification of non-Hermitian systems in Altland-Zirnbauer class $\mathrm{A}$ is $\mathbb{Z}[31]$.)

In the presence of crystalline inversion symmetry, $w_{1 \mathrm{D}}$ mod 2 can be expressed purely in terms of inversion eigenvalues at inversion-symmetric momenta. For this, first note that for non-Hermitian systems, there are two ways of implementing inversion symmetry: (i) "standard" inversion symmetry implies

$$
\mathcal{I H}(k) \mathcal{I}^{\dagger}=\mathcal{H}(-k)
$$

for some unitary matrix $\mathcal{I}$. (ii) "Pseudo"inversion symmetry implies

$$
\mathcal{I H}(k) \mathcal{I}^{\dagger}=\mathcal{H}^{\dagger}(-k) .
$$

In both cases, $\mathcal{I}^{2}=\mathbb{1}$. The two options are equivalent in the Hermitian case. For systems with a skin effect, pseudoinversion is the relevant symmetry. With standard inversion symmetry,

$$
w_{1 \mathrm{D}}=\int_{0}^{2 \pi} \frac{d k}{2 \pi i} \frac{d}{d k} \ln \operatorname{det} \mathcal{H}(-k)=-w_{1 \mathrm{D}},
$$

so that the only admissible winding number is $w_{1 \mathrm{D}}=0$. And indeed, the Bloch Hamiltonian in Eq. (2) satisfies pseudoinversion: $\mathcal{H}(k)=\mathcal{H}^{\dagger}(-k)$, so that $\mathcal{I}=1$.

Let us therefore consider a general 1D non-Hermitian Bloch Hamiltonian $\mathcal{H}(k)$ that satisfies Eq. (5). Its Hermitian double is given by

$$
\overline{\mathcal{H}}(k)=\left(\begin{array}{cc}
0 & \mathcal{H}(k) \\
\mathcal{H}^{\dagger}(k) & 0
\end{array}\right) .
$$

The presence of a point gap of $\mathcal{H}(k)$ around $E=0$ translates into a gapped spectrum of $\overline{\mathcal{H}}(k)$. By construction, $\overline{\mathcal{H}}(k)$ furthermore enjoys a chiral (sublattice) symmetry:

$$
\overline{\mathcal{C}} \overline{\mathcal{H}}(k) \overline{\mathcal{C}}^{\dagger}=-\overline{\mathcal{H}}(k), \quad \overline{\mathcal{C}}=\left(\begin{array}{cc}
\mathbb{1} & 0 \\
0 & -\mathbb{1}
\end{array}\right),
$$

positioning the Hermitian double in symmetry class AIII of the Altland-Zirnbauer classification [on the other hand, the non-Hermitian Hamiltonian $\mathcal{H}(k)$ has no internal symmetries and so it lies in class A]. The quantity $w_{1 \mathrm{D}}$ is then merely the winding number of 1D Hermitian systems with chiral symmetry, and as such it counts the number of protected zero-energy edge states. Moreover, the pseudoinversion symmetry of $\mathcal{H}(k)$ implies a standard inversion symmetry for $\overline{\mathcal{H}}(k)$ :

$$
\overline{\mathcal{I}} \overline{\mathcal{H}}(k) \overline{\mathcal{I}}^{\dagger}=\overline{\mathcal{H}}(-k), \quad \overline{\mathcal{I}}=\left(\begin{array}{cc}
0 & \mathcal{I} \\
\mathcal{I} & 0
\end{array}\right) .
$$

(Note that $\overline{\mathcal{I}}$ and $\overline{\mathcal{C}}$ anticommute if the non-Hermitian Hamiltonian has pseudoinversion, whereas for standard inversion symmetry $\overline{\mathcal{I}}$ and $\overline{\mathcal{C}}$ commute and so they preclude any symmetry-indicated band inversions.) Correspondingly, the eigenstates of $\overline{\mathcal{H}}(k)$ can be chosen as eigenstates of $\overline{\mathcal{I}}$ at the inversion-symmetric momenta $k=0, \pi$. It is well known $[42,44]$ that $w_{1 \mathrm{D}}$ is related to the Zak phase $\gamma$ of $\overline{\mathcal{H}}(k)$ via

$$
w_{1 \mathrm{D}} \bmod 2=\gamma / \pi \text {. }
$$

Furthermore, Ref. [45] showed that the $\mathbb{Z}_{2}$-valued Zak phase is constrained by the relation

$$
\gamma=\frac{\pi}{2} \sum_{k^{*} \in \mathcal{I} \text { SMs }} e^{i k^{*}} \operatorname{Tr}\left[\overline{\mathcal{I}} \bar{P}\left(k^{*}\right)\right] \bmod 2 \pi,
$$

where $\bar{P}(k)$ is the projector onto the occupied subspace of $\overline{\mathcal{H}}(k)$, and the inversion-symmetric momenta are given by $\mathcal{I S M s}=\{0, \pi\}$. Equations (10) and (11) provide an example of a symmetry indicator formula for a non-Hermitian topological invariant.

$3 D$ winding number. We next formulate symmetry indicator invariants for 3D point-gapped non-Hermitian Hamiltonians $\mathcal{H}(\boldsymbol{k})$ that are characterized by the 3D winding number $[30,31,46]$

$$
w_{3 \mathrm{D}}=-\sum_{i j k} \int_{\mathrm{BZ}} \frac{d^{3} \boldsymbol{k}}{24 \pi^{2}} \epsilon_{i j k} \operatorname{Tr}\left[Q_{i}(\boldsymbol{k}) Q_{j}(\boldsymbol{k}) Q_{k}(\boldsymbol{k})\right] \in \mathbb{Z},
$$

where $Q_{j}(\boldsymbol{k})=\mathcal{H}(\boldsymbol{k})^{-1} \partial_{k_{j}} \mathcal{H}(\boldsymbol{k}), \quad j=x, y, z, \quad$ and $\quad \mathrm{BZ}=$ $[0,2 \pi]^{3}$ denotes the 3D Brillouin zone. A nontrivial $w_{3 \mathrm{D}}$ has been identified with the presence of anomalous non-Hermitian surface states in Ref. [47], giving rise to exceptional topological insulators (ETIs). Again, the Hermitian double $\overline{\mathcal{H}}(\boldsymbol{k})$, defined in analogy to Eq. (7), describes a gapped 3D system in Altland-Zirnbauer class AIII. The invariant $w_{3 \mathrm{D}}$ then counts the number of protected (twofold) Dirac cone surface states [48].

To make contact with the $1 \mathrm{D}$ case, we begin by discussing systems with inversion symmetry. In analogy to Eq. (6), standard inversion symmetry implies $w_{3 \mathrm{D}}=0$, prompting us to focus on pseudoinversion [Eq. (5)]. Defining the inversion operator $\overline{\mathcal{I}}$ of the Hermitian double as in Eq. (9), we now prove

$$
w_{3 \mathrm{D}} \bmod 2=v_{2},
$$

where the $\mathbb{Z}_{2}$-valued symmetry indicator $v_{2}$ is defined by

$$
\nu_{2}=\frac{1}{4} \sum_{\boldsymbol{k}^{*} \in \mathcal{I} \mathrm{SMs}} \operatorname{Tr}\left[\overline{\mathcal{I}} \bar{P}\left(\boldsymbol{k}^{*}\right)\right] \bmod 2 .
$$

Here, $\bar{P}(\boldsymbol{k})$ is again the projector onto the occupied subspace of the Hermitian double $\overline{\mathcal{H}}(\boldsymbol{k})$, while the inversion-symmetric momenta are now drawn from

$$
\begin{aligned}
\mathcal{I S M s}= & (0,0,0),(0, \pi, 0),(\pi, 0,0),(\pi, \pi, 0), \\
& (0,0, \pi),(0, \pi, \pi),(\pi, 0, \pi),(\pi, \pi, \pi)\} .
\end{aligned}
$$

To derive the relation, we begin by noting that $\nu_{2}=1$ is precisely the condition for a gapped, inversion-symmetric Hermitian Hamiltonian (for now without chiral symmetry) to be a symmetry-indicated axion insulator (AXI) [49-51]. AXIs are (time-reversal broken) higher-order topological insulators that host one-dimensional unidirectional (chiral) hinge states 
(a)

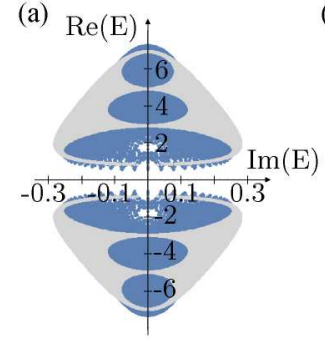

(c)

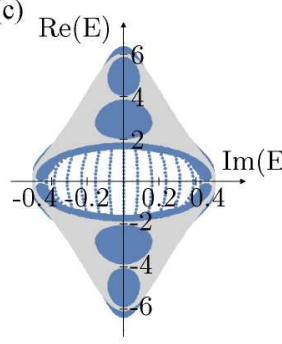

(b)

(d)
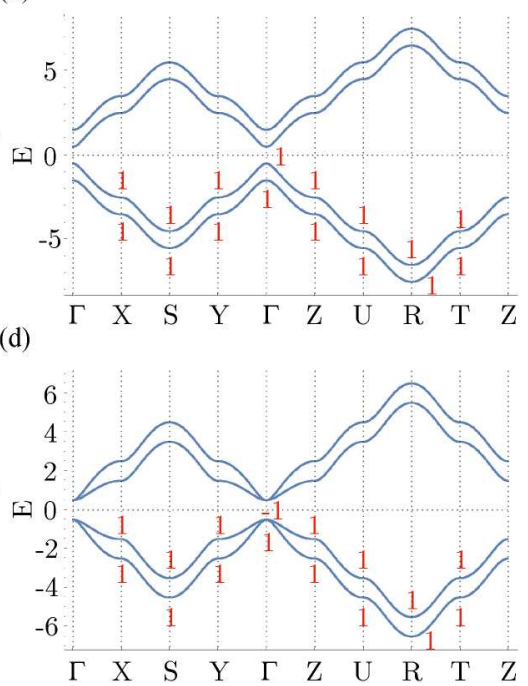

FIG. 1. Inversion symmetry indicator for $w_{3 \mathrm{D}}$. (a),(c) Bulk (gray) and surface (blue) spectra in the complex plane for a non-Hermitian topological insulator with $w_{3 \mathrm{D}}=0$ (a) and $w_{3 \mathrm{D}}=1$ (c) [43]. (b),(d) Band structure of the corresponding Hermitian doubles along high-symmetry lines of the Brillouin zone. All bands are twofolddegenerate, and each pair has equal inversion eigenvalues at the inversion-symmetric momenta (the inversion eigenvalues of the occupied bands are shown in red). By Eq. (13), the presence (absence) of a double band inversion in (d) [in (b)] indicates the presence (absence) of anomalous non-Hermitian surface states in (c) [in (a)].

when cut into inversion-symmetric geometries. Their surfaces each host a gapped Dirac cone as a remnant of the bulk double band inversion (indicated by $v_{2}=1$ ). In fact, it is instructive to regard the hinge states of an AXI as domain wall bound states in the surface Dirac mass [52]. To prove Eq. (13), we then only need to show that the surfaces of an AXI respecting inversion and chiral symmetry must remain gapless, because $w_{3 D}$ counts the number of protected surface Dirac cones of the Hermitian double. But this must be the case, because the unidirectional dispersion of chiral hinge states, whose presence is implied by any inversion-preserving surface gap, violates chiral symmetry as defined in Eq. (8). (See Fig. 1.)

To drive this point further, consider the Dirac surface theory of an AXI with chiral symmetry (where the surface normal is chosen to lie along the $z$-direction) [39],

$$
\overline{\mathcal{H}}_{\mathrm{D}}\left(k_{x}, k_{y}\right)=k_{x} \sigma_{x}+k_{y} \sigma_{y},
$$

where $\sigma_{x, y, z}$ is a set of Pauli matrices. Here, chiral symmetry is represented by $\overline{\mathcal{C}}_{\mathrm{D}}=\sigma_{z}$. The only mass term, multiplying $\sigma_{z}$, does not anticommute with chiral symmetry and is therefore disallowed. Generalizing this, we conclude that all surfaces remain gapless and host a single Dirac cone, vindicating Eq. (13).

It is possible to calculate the symmetry indicator $\nu_{2}$ without explicitly constructing the Hermitian double. For this, we utilize the singular-value decomposition [53], which was previously fruitfully applied in the context of non-Hermitian topological systems [54]:

$$
\mathcal{H}(\boldsymbol{k})=U(\boldsymbol{k}) \Sigma(\boldsymbol{k}) V(\boldsymbol{k})^{\dagger},
$$

where $U(\boldsymbol{k}), V(\boldsymbol{k})$ are unitary matrices of the same dimension as $H(\boldsymbol{k})$, and $\Sigma(\boldsymbol{k})$ is a diagonal matrix with non-negative entries. We have that

$$
\mathcal{H}(\boldsymbol{k}) V(\boldsymbol{k})=U(\boldsymbol{k}) \Sigma(\boldsymbol{k}), \quad \mathcal{H}(\boldsymbol{k})^{\dagger} U(\boldsymbol{k})=V(\boldsymbol{k}) \Sigma(\boldsymbol{k})
$$

implying that the matrix

$$
S(\boldsymbol{k})=\frac{1}{\sqrt{2}}\left[\begin{array}{cc}
U(\boldsymbol{k}) & U(\boldsymbol{k}) \\
V(\boldsymbol{k}) & -V(\boldsymbol{k})
\end{array}\right]
$$

diagonalizes the Hermitian double $\overline{\mathcal{H}}(\boldsymbol{k})$ :

$$
S(\boldsymbol{k})^{\dagger} \overline{\mathcal{H}}(\boldsymbol{k}) S(\boldsymbol{k})=\left[\begin{array}{cc}
\Sigma(\boldsymbol{k}) & 0 \\
0 & -\Sigma(\boldsymbol{k})
\end{array}\right] .
$$

We therefore find

$$
v_{2}=-\frac{1}{8} \sum_{\boldsymbol{k}^{*} \in \mathcal{I S M s}} \operatorname{Tr}\left[V(\boldsymbol{k})^{\dagger} \mathcal{I} U(\boldsymbol{k})+U(\boldsymbol{k})^{\dagger} \mathcal{I} V(\boldsymbol{k})\right] \bmod 2 .
$$

Chiral Chern-Simons form. We now turn to 3D nonHermitian insulators in symmetry class AII. This class is characterized by a standard time-reversal symmetry, so that the non-Hermitian Bloch Hamiltonian and the Hermitian double satisfy

$$
\begin{aligned}
& \mathcal{T} \mathcal{H}(\boldsymbol{k}) \mathcal{T}^{\dagger}=\mathcal{H}(-\boldsymbol{k}), \\
& \overline{\mathcal{T}} \overline{\mathcal{H}}(\boldsymbol{k}) \overline{\mathcal{T}}^{\dagger}=\overline{\mathcal{H}}(-\boldsymbol{k}), \quad \overline{\mathcal{T}}=\left(\begin{array}{ll}
\mathcal{T} & 0 \\
0 & \mathcal{T}
\end{array}\right)
\end{aligned}
$$

for some antiunitary operator $\mathcal{T}$. Together with the 3D version of Eq. (8), we then have that time-reversal and chiral symmetry commute: $[\overline{\mathcal{T}}, \overline{\mathcal{C}}]=0$, locating the Hermitian double in Altland-Zirnbauer class CII. As previously explained, Eq. (22) necessitates $w_{3 \mathrm{D}}=0$. Nevertheless, the classification of non-Hermitian systems in Altland-Zirnbauer class AII (corresponding to a Hermitian double in class CII) is given by $\mathbb{Z}_{2}$ [31]; it is indicated by the chiral Chern-Simons form $\mathrm{CS}_{3}$ whose evaluation requires a smooth gauge of Bloch states over the Brillouin zone [42]. Note that the ETI with $w_{3 \mathrm{D}}=1$ is adiabatically related to a $k_{z}$-indexed pumping cycle of a $2 \mathrm{D}$ integer quantum Hall effect (with Chern number 1) around the complex point gap [47]. Then, appealing to the correspondence between the $\mathbb{Z}$-classified integer quantum Hall effect in class $\mathrm{A}$ and the $\mathbb{Z}_{2}$-classified quantum spin Hall effect in class AII, it is straightforward to construct a representative of a nontrivial point-gapped phase in class AII: we take two time-reversal related copies of the ETI and form the tensor sum

$$
\mathcal{H}(\boldsymbol{k})=\left(\begin{array}{cc}
\mathcal{H}_{\mathrm{ETI}}(\boldsymbol{k}) & 0 \\
0 & T^{\dagger} \mathcal{H}_{\mathrm{ETI}}(-\boldsymbol{k}) T
\end{array}\right),
$$

where $T$ is an antiunitary operator that squares to -1 . The complex spectrum of $\mathcal{H}(\boldsymbol{k})$ can develop a real line gap under the addition of symmetry-allowed perturbations. However, we will see that the surface spectrum necessarily remains gapless (in that there is no line gap). The non-Hermitian Hamiltonian satisfies Eq. (22) with

$$
\mathcal{T}=\left(\begin{array}{cc}
0 & T \\
T^{\dagger} & 0
\end{array}\right)
$$


Because time-reversal symmetry commutes with pseudoinversion symmetry, the inversion eigenvalues of the Hermitian double form two copies of the inversion eigenvalues of an AXI and we obtain $v_{4}=2$, where $v_{4}$ is the symmetry indicator,

$$
v_{4}=\frac{1}{4} \sum_{\boldsymbol{k}^{*} \in \mathcal{I S M s}} \operatorname{Tr}\left[\overline{\mathcal{I}} \bar{P}\left(\boldsymbol{k}^{*}\right)\right] \bmod 4,
$$

associated with the simultaneous presence of inversion and time-reversal symmetry. Furthermore, we will show that the surfaces of $\mathcal{H}(\boldsymbol{k})$ are gapless and anomalous, in that they form two time-reversal related copies of the anomalous surface states of an ETI. These observations prompt us to posit

$$
\mathrm{CS}_{3} \bmod 2=\frac{1}{2} v_{4} \bmod 2,
$$

where

$$
\begin{aligned}
\mathrm{CS}_{3}= & -\sum_{i j k} \int_{\mathrm{BZ}} \frac{d^{3} \boldsymbol{k}}{8 \pi^{2}} \epsilon_{i j k} \operatorname{Tr}\left[A_{i}(\boldsymbol{k}) \partial_{k_{j}} A_{k}(\boldsymbol{k})\right. \\
& \left.+\frac{2}{3} A_{i}(\boldsymbol{k}) A_{j}(\boldsymbol{k}) A_{k}(\boldsymbol{k})\right]
\end{aligned}
$$

is the chiral Chern-Simons form, the invariant for Hermitian insulators in class CII [31,42]. A nontrivial $\mathrm{CS}_{3}$ corresponds to a stable pair of Dirac cones in the surface spectrum of the Hermitian double [48], whose presence is heralded by $v_{4}=2$ (recall that we have previously shown that the surfaces of an AXI host a single Dirac cone as long as chiral symmetry is enforced). Here, we have made use of the non-Abelian, matrix-valued Berry connection

$$
\left[A_{i}(\boldsymbol{k})\right]^{m n}=\left\langle u^{m}(\boldsymbol{k})\left|\partial_{k_{i}}\right| u^{n}(\boldsymbol{k})\right\rangle,
$$

which is defined in terms of the occupied (negative-energy) Bloch eigenstates $\left|u^{n}(\boldsymbol{k})\right\rangle$ of $\overline{\mathcal{H}}(\boldsymbol{k})$. We note that Eq. (27) is gauge-dependent and needs to be supplemented by the gauge condition

$$
\int_{\partial \mathrm{BZ}_{1 / 2}} d^{2} \boldsymbol{k} \epsilon_{i j} \operatorname{Tr}\left\{\left[X(\boldsymbol{k}) \partial_{k_{i}} X^{\dagger}(\boldsymbol{k})\right]\left[X(\boldsymbol{k}) \partial_{k_{j}} X^{\dagger}(\boldsymbol{k})\right]\right\}=0,
$$

where

$$
X(\boldsymbol{k})=\left[\left|u^{1}(\boldsymbol{k})\right\rangle, \ldots,\left|u^{N}(\boldsymbol{k})\right\rangle,\left|v^{1}(\boldsymbol{k})\right\rangle, \ldots,\left|v^{N}(\boldsymbol{k})\right\rangle\right]
$$

is the unitary matrix of occupied and unoccupied eigenstates of $\overline{\mathcal{H}}(\boldsymbol{k})$, respectively. Evidently, the integral in Eq. (27) and its associated gauge condition in Eq. (29) are sufficiently involved as to make an expression in terms of symmetry indicators highly desirable.
For Eq. (26) to be well-defined, $v_{4}=1,3$ should not be admissible. And indeed, by the Fu-Kane criterion [36], these values indicate an odd number of double-band inversions in the bulk of the Hermitian double [ $2(2 n+1), n \in \mathbb{Z}$, inversion eigenvalues of the valence manifold switch signs with respect to the atomic limit], however such a scenario is disallowed in symmetry class CII [48].

Finally, we discuss the surface physics of $\mathcal{H}(\boldsymbol{k})$ in Eq. (23). In the unperturbed case, Eq. (23), each ETI contributes a single exceptional point to the complex surface spectrum. We can model the surface with $z$-normal by the Dirac Hamiltonian

$$
\mathcal{H}_{\mathrm{D}}\left(k_{x}, k_{y}\right)=\left(k_{x}+i c_{x}\right) \sigma_{x}+\left(k_{y}+i c_{y}\right) \sigma_{y}+i c_{z} \sigma_{z},
$$

where $c_{x, y, z}$ are real numbers and $\sigma_{x, y, z}$ is a set of Pauli matrices. This Hamiltonian satisfies standard time-reversal symmetry with $\mathcal{T}=i \sigma_{y} K$, where $K$ denotes complex conjugation. For generic values of $c_{x, y, z}$, there are two exceptional points in the spectrum of $\mathcal{H}_{\mathrm{D}}(k)$. These may morph into an exceptional loop for $c_{x}=c_{y}=0$, or into a Hermitian Dirac cone for $c_{x}=c_{y}=c_{z}=0$, but since $\mathcal{H}_{\mathrm{D}}(k)$ exhausts all terms allowed by symmetry to linear order in $k$ (up to unitary basis transformations and terms multiplying the identity matrix), they can never annihilate. We therefore conclude that the surface must host two exceptional points. In the Supplemental Material [55], we discuss this case and the pseudo-timereversal symmetric (reciprocal [56]) case with $v_{4}=2$. There, we also present tight-binding models for all phases discussed here.

Discussion. We have derived symmetry indicator invariants for $w_{1 \mathrm{D}}$ and $w_{3 \mathrm{D}}$, which classify point-gapped non-Hermitian insulators in Altland-Zirnbauer symmetry class A. Furthermore, we showed that non-Hermitian time-reversal symmetric topological insulators can be identified using symmetry indicators without the need for a smooth Bloch gauge. To connect non-Hermitian topological invariants with the symmetry indicators of the Hermitian double, our strategy was to study the effect of chiral symmetry on Hermitian topological crystalline insulators. Interestingly, the introduction of chiral symmetry changes the bulk-boundary correspondence of these insulators while leaving their symmetry indicators intact. This approach can be straightforwardly generalized to other symmetry classes.

Note added. While preparing this manuscript, we became aware of a recent related work [57], which also investigates non-Hermitian symmetry indicators, but it focuses on bulk exceptional points and lines in two and three dimensions.

Acknowledgments. This project has received funding from the European Research Council (ERC) under the European Union's Horizon 2020 research and innovation programme (ERC-StG-Neupert-757867-PARATOP).
[1] D. Leykam, K. Y. Bliokh, C. Huang, Y. D. Chong, and F. Nori, Phys. Rev. Lett. 118, 040401 (2017).

[2] H. Shen, B. Zhen, and L. Fu, Phys. Rev. Lett. 120, 146402 (2018).

[3] S. Lieu, Phys. Rev. B 97, 045106 (2018).
[4] Z.-Y. Ge, Y.-R. Zhang, T. Liu, S.-W. Li, H. Fan, and F. Nori, Phys. Rev. B 100, 054105 (2019).

[5] J. Y. Lee, J. Ahn, H. Zhou, and A. Vishwanath, Phys. Rev. Lett. 123, 206404 (2019).

[6] Y. Xiong, J. Phys. Commun. 2, 035043 (2018). 
[7] F. K. Kunst, E. Edvardsson, J. C. Budich, and E. J. Bergholtz, Phys. Rev. Lett. 121, 026808 (2018).

[8] S. Yao and Z. Wang, Phys. Rev. Lett. 121, 086803 (2018).

[9] D. S. Borgnia, A. J. Kruchkov, and R.-J. Slager, Phys. Rev. Lett. 124, 056802 (2020).

[10] A. Stegmaier, S. Imhof, T. Helbig, T. Hofmann, C. H. Lee, M. Kremer, A. Fritzsche, T. Feichtner, S. Klembt, S. Höfling et al., arXiv:2011.14836.

[11] Z. Yang, K. Zhang, C. Fang, and J. Hu, Phys. Rev. Lett. 125, 226402 (2020).

[12] Z. Yang, A. P. Schnyder, J. Hu, and C.-K. Chiu, Phys. Rev. Lett. 126, 086401 (2021).

[13] N. Hatano and D. R. Nelson, Phys. Rev. Lett. 77, 570 (1996).

[14] N. Hatano and D. R. Nelson, Phys. Rev. B 56, 8651 (1997).

[15] N. Hatano and D. R. Nelson, Phys. Rev. B 58, 8384 (1998).

[16] T. E. Lee, Phys. Rev. Lett. 116, 133903 (2016).

[17] V. M. Martinez Alvarez, J. E. Barrios Vargas, and L. E. F. Foa Torres, Phys. Rev. B 97, 121401(R) (2018).

[18] S. Longhi, Phys. Rev. Research 1, 023013 (2019).

[19] F. Song, S. Yao, and Z. Wang, Phys. Rev. Lett. 123, 170401 (2019).

[20] C. H. Lee and R. Thomale, Phys. Rev. B 99, 201103(R) (2019).

[21] N. Okuma, K. Kawabata, K. Shiozaki, and M. Sato, Phys. Rev. Lett. 124, 086801 (2020).

[22] E. Lee, H. Lee, and B.-J. Yang, Phys. Rev. B 101, 121109(R) (2020).

[23] S. Mu, C. H. Lee, L. Li, and J. Gong, Phys. Rev. B 102, 081115(R) (2020).

[24] T. Helbig, T. Hofmann, S. Imhof, M. Abdelghany, T. Kiessling, L. W. Molenkamp, C. H. Lee, A. Szameit, M. Greiter, and R. Thomale, Nat. Phys. 16, 747 (2020).

[25] T. Hofmann, T. Helbig, F. Schindler, N. Salgo, M. Brzezińska, M. Greiter, T. Kiessling, D. Wolf, A. Vollhardt, A. Kabaši, C. H. Lee, A. Bilušić, R. Thomale, and T. Neupert, Phys. Rev. Research 2, 023265 (2020).

[26] T. Yoshida, T. Mizoguchi, and Y. Hatsugai, Phys. Rev. Research 2, 022062(R) (2020).

[27] L. Li, C. H. Lee, S. Mu, and J. Gong, Nat. Commun. 11, 5491 (2020).

[28] A. Ghatak, M. Brandenbourger, J. van Wezel, and C. Coulais, Proc. Natl. Acad. Sci. (USA) 117, 29561 (2020).

[29] S. Weidemann, M. Kremer, T. Helbig, T. Hofmann, A. Stegmaier, M. Greiter, R. Thomale, and A. Szameit, Science 368, 311 (2020).

[30] Z. Gong, Y. Ashida, K. Kawabata, K. Takasan, S. Higashikawa, and M. Ueda, Phys. Rev. X 8, 031079 (2018).

[31] K. Kawabata, K. Shiozaki, M. Ueda, and M. Sato, Phys. Rev. X 9, 041015 (2019).

[32] C.-H. Liu, H. Jiang, and S. Chen, Phys. Rev. B 99, 125103 (2019).
[33] C.-H. Liu and S. Chen, Phys. Rev. B 100, 144106 (2019).

[34] H. Zhou and J. Y. Lee, Phys. Rev. B 99, 235112 (2019).

[35] K. Zhang, Z. Yang, and C. Fang, Phys. Rev. Lett. 125, 126402 (2020).

[36] L. Fu and C. L. Kane, Phys. Rev. B 76, 045302 (2007).

[37] J. Kruthoff, J. de Boer, J. van Wezel, C. L. Kane, and R.-J. Slager, Phys. Rev. X 7, 041069 (2017).

[38] H. C. Po, A. Vishwanath, and H. Watanabe, Nat. Commun. 8, 50 (2017)

[39] E. Khalaf, H. C. Po, A. Vishwanath, and H. Watanabe, Phys. Rev. X 8, 031070 (2018).

[40] B. Bradlyn, L. Elcoro, J. Cano, M. G. Vergniory, Z. Wang, C. Felser, M. I. Aroyo, and B. A. Bernevig, Nature 547, 298 (2017).

[41] S. Ono and H. Watanabe, Phys. Rev. B 98, 115150 (2018).

[42] C.-K. Chiu, J. C. Y. Teo, A. P. Schnyder, and S. Ryu, Rev. Mod. Phys. 88, 035005 (2016).

[43] We use the Hamiltonian introduced in Eq. (1) of Ref. [47] with $M=4$ (trivial) and $M=3$ (nontrivial), $\lambda=1, \delta=0.5$, $B=0.2$, and $\alpha=\pi / 2$.

[44] T. Neupert and F. Schindler, Topological crystalline insulators, in Topological Matter: Lectures from the Topological Matter School 2017, edited by D. Bercioux, J. Cayssol, M. G. Vergniory, and M. Reyes Calvo (Springer International, Cham, 2018), pp. 31-61.

[45] A. Alexandradinata, X. Dai, and B. A. Bernevig, Phys. Rev. B 89, 155114 (2014).

[46] A. Ghatak and T. Das, J. Phys.: Condens. Matter 31, 263001 (2019).

[47] M. M. Denner, A. Skurativska, F. Schindler, M. H. Fischer, R. Thomale, T. Bzdušek, and T. Neupert, arXiv:2008.01090.

[48] A. P. Schnyder, S. Ryu, A. Furusaki, and A. W. W. Ludwig, Phys. Rev. B 78, 195125 (2008).

[49] A. M. Turner, Y. Zhang, and A. Vishwanath, Phys. Rev. B 82, 241102(R) (2010).

[50] N. Varnava and D. Vanderbilt, Phys. Rev. B 98, 245117 (2018).

[51] B. J. Wieder and B. A. Bernevig, arXiv:1810.02373.

[52] B. J. Wieder, Z. Wang, J. Cano, X. Dai, L. M. Schoop, B. Bradlyn, and B. A. Bernevig, Nat. Commun. 11, 627 (2020).

[53] Similarly, one may leverage the singular value decomposition to express Eq. (11) without reference to the Hermitian double.

[54] L. Herviou, J. H. Bardarson, and N. Regnault, Phys. Rev. A 99, 052118 (2019).

[55] See Supplemental Material at http://link.aps.org/supplemental/ 10.1103/PhysRevB.103.L201114 for model Hamiltonians and further derivations.

[56] K. Kawabata, N. Okuma, and M. Sato, Phys. Rev. B 101, 195147 (2020).

[57] R. Okugawa, R. Takahashi, and K. Yokomizo, Phys. Rev. B 103, 205205 (2021). 